\title{
BEYOND GÖLLNITZ' THEOREM I: A BIJECTIVE APPROACH
}

\author{
ISAAC KONAN
}

\begin{abstract}
In 2003, Alladi, Andrews and Berkovich proved an identity for partitions where parts occur in eleven colors: four primary colors, six secondary colors, and one quaternary color. Their work answered a longstanding question of how to go beyond a classical theorem of Göllnitz, which uses three primary and three secondary colors. Their main tool was a deep and difficult four parameter $q$-series identity. In this paper we take a different approach. Instead of adding an eleventh quaternary color, we introduce forbidden patterns and give a bijective proof of a ten-colored partition identity lying beyond Göllnitz' theorem. Using a second bijection, we show that our identity is equivalent to the identity of Alladi, Andrews, and Berkovich. From a combinatorial viewpoint, the use of forbidden patterns is more natural and leads to a simpler formulation. In fact, in Part II of this series we will show how our method can be used to go beyond Göllnitz' theorem to any number of primary colors.
\end{abstract}

\section{Introduction and Statements of Results}

1.1. History. A partition of a positive integer $n$ is a non-increasing sequence of positive integers whose sum is equal to $n$. For example, the partitions of 7 are

$$
\begin{gathered}
(7),(6,1),(5,2),(5,1,1),(4,3),(4,2,1),(4,1,1,1),(3,3,1),(3,2,2),(3,2,1,1), \\
(3,1,1,1,1),(2,2,2,1),(2,2,1,1,1),(2,1,1,1,1,1) \text { and }(1,1,1,1,1,1,1) .
\end{gathered}
$$

The study of partition identities has a long history, dating back to Euler's proof that there are as many partitions of $n$ into distinct parts as partitions of $n$ into odd parts. The corresponding identity is

$$
(-q ; q)_{\infty}=\frac{1}{\left(q ; q^{2}\right)_{\infty}}
$$

where

$$
(x ; q)_{m}=\prod_{k=0}^{m-1}\left(1-x q^{k}\right),
$$

for any $m \in \mathbb{Z}_{\geq 0} \cup\{\infty\}$ and $x, q$ such that $|q|<1$.

One of the most important identities in the theory of partitions is Schur's theorem [9].

Theorem 1.1 (Schur 1926). For any positive integer n, the number of partitions of $n$ into distinct parts congruent to $\pm 1 \bmod 3$ is equal to the number of partitions of $n$ where parts differ by at least three and multiples of three differ by at least six.

There have been a number of proofs of Schur's result over the years, including a $q$-difference equation proof of Andrews [4] and a simple bijective proof of Bressoud [6].

Another important identity is Göllnitz' theorem [7]. 
Theorem 1.2 (Göllnitz 1967). For any positive integer n, the number of partitions of $n$ into distinct parts congruent to 2, 4,5 $\bmod 6$ is equal to the number of partitions of $n$ into parts different from 1 and 3 , and where parts differ by at least six with equality only if parts are congruent to $2,4,5 \bmod 6$.

Like Schur's theorem, Göllnitz's identity can be proved using q-difference equations [5] and an elegant Bressoud-style bijection [8, 10].

Seminal work of Alladi, Andrews, and Gordon in the 90's showed how the theorems of Schur and Göllnitz emerge from more general results on colored partitions [2].

In the case of Schur's theorem, we consider parts in three colors $\{a, b, a b\}$ and order them as follows:

$$
1_{a b}<1_{a}<1_{b}<2_{a b}<2_{a}<2_{b}<3_{a b}<\cdots
$$

We then consider the partitions with colored parts different from $1_{a b}$ and satisfying the minimal difference conditions in the table

\begin{tabular}{|c|cc|c|}
\hline$\lambda_{i} \backslash^{\lambda_{i+1}}$ & $a$ & $b$ & $a b$ \\
\hline$a$ & 1 & 2 & 1 \\
$b$ & 1 & 1 & 1 \\
\hline$a b$ & 2 & 2 & 2 \\
\hline
\end{tabular}

Here, the part $\lambda_{i}$ with color in the row and the part $\lambda_{i+1}$ with color in the column differ by at least the corresponding entry in the table. An example of such a partition is $\left(7_{a b}, 5_{b}, 4_{a}, 3_{a b}, 1_{b}\right)$. The Alladi-Gordon refinement of Schur's partition theorem [3] is stated as follows:

Theorem 1.3. Let $u, v, n$ be non-negative integers. Denote by $A(u, v, n)$ the number of partitions of $n$ into $u$ distncts parts with color a and $v$ distinct parts with color $b$, and denote by $B(u, v, n)$ the number of partitions of $n$ satisfying the conditions in (1.3), with $u$ parts with color $a$ or $a b$, and $v$ parts with color $b$ or $a b$. We then have $A(u, v, n)=B(u, v, n)$ and the identity

$$
\sum_{u, v, n \geq 0} B(u, v, n) a^{u} b^{v} q^{n}=\sum_{u, v, n \geq 0} A(u, v, n) a^{u} b^{v} q^{n}=(-a q ; q)_{\infty}(-b q ; q)_{\infty} .
$$

Note that a transformation implies Schur's theorem :

$$
\left\{\begin{array}{l}
\text { dilation : } \quad q \quad \mapsto q^{3} \\
\text { translations : } a, b
\end{array}\right.
$$

In fact, the minimal difference conditions given in (1.3) give after these transformations the minimal differences in Schur's theorem.

In the case of Göllnitz' theorem, we consider parts that occur in six colors $\{a, b, c, a b, a b, b c\}$ with the order

$$
1_{a b}<1_{a c}<1_{a}<1_{b c}<1_{b}<1_{c}<2_{a b}<2_{a c}<2_{a}<2_{b c}<2_{b}<2_{c}<3_{a b}<\cdots,
$$


and the partitions with colored parts different from $1_{a b}, 1_{a c}, 1_{b c}$ and satisfying the minimal difference conditions in

\begin{tabular}{|c|ccc|ccc|}
\hline$\lambda_{i}{ }^{\lambda_{i+1}}$ & $a$ & $b$ & $c$ & $a b$ & $a c$ & $b c$ \\
\hline$a$ & 1 & 2 & 2 & 1 & 1 & 2 \\
$b$ & 1 & 1 & 2 & 1 & 1 & 1 \\
$c$ & 1 & 1 & 1 & 1 & 1 & 1 \\
\hline$a b$ & 2 & 2 & 2 & 2 & 2 & 2 \\
$a c$ & 2 & 2 & 2 & 1 & 2 & 2 \\
$b c$ & 1 & 2 & 2 & 1 & 1 & 2 \\
\hline
\end{tabular}

The Alladi-Andrews-Gordon refinement of Göllnitz's partition theorem can be stated as follows:

Theorem 1.4. Let $u, v, w, n$ be non-negative integers. Denote by $A(u, v, w, n)$ the number of partitions of $n$ into $u$ distncts parts with color $a, v$ distinct parts with color $b$ and $w$ distinct parts with color $c$, and denote by $B(u, v, w, n)$ the number of partitions of $n$ satisfying the conditions in (1.7), with $u$ parts with color $a, a b$ or ac, $v$ parts with color $b, a b$ or bc and $w$ parts with color $c$, ac or bc. We then have $A(u, v, w, n)=B(u, v, w, n)$ and the identity

$\sum_{u, v, w, n \geq 0} B(u, v, w, n) a^{u} b^{v} c^{w} q^{n}=\sum_{u, v, w, n \geq 0} A(u, v, w, n) a^{u} b^{v} c^{w} q^{n}=(-a q ; q)_{\infty}(-b q ; q)_{\infty}(-c q ; q)_{\infty}$.

Note that a transformation implies Göllnitz' theorem :

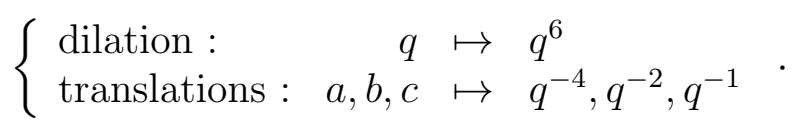

Observe that while Schur's theorem is not a direct corollary of Göllnitz' theorem, Theorem 1.3 is implied by Theorem $\mathbf{1 . 4}$ by setting $c=0$. Therefore Göllnitz' theorem may be viewed as a level higher than Schur's theorem, since it requires three primary colors instead of two.

Following the work of Alladi, Andrews, and Gordon, it was an open problem to find a partition identity beyond Göllnitz' theorem, in the sense that it would arise from four primary colors. This was famously solved by Alladi, Andrews, and Berkovich [1. To describe their result, we consider parts that occur in eleven colors $\{a, b, c, d, a b, a c, a d, b c, b d, c d, a b c d\}$ and ordered as follows:

$$
1_{a b c d}<1_{a b}<1_{a c}<1_{a d}<1_{a}<1_{b c}<1_{b d}<1_{b}<1_{c d}<1_{c}<1_{d}<2_{a b c d}<\cdots
$$


Let us consider the partitions with the size of the secondary parts greater than one and satisfying the minimal difference conditions in

\begin{tabular}{|c|cccc|ccc|cc|c|}
\hline$\lambda_{i} \backslash^{\lambda_{i+1}}$ & $a b$ & $a c$ & $a d$ & $a$ & $b c$ & $b d$ & $b$ & $c d$ & $c$ & $d$ \\
\hline$a b$ & 2 & 2 & 2 & 2 & 2 & 2 & 2 & 2 & 2 & 2 \\
$a c$ & 1 & 2 & 2 & 2 & 2 & 2 & 2 & 2 & 2 & 2 \\
$a d$ & 1 & 1 & 2 & 2 & 2 & 2 & 2 & 2 & 2 & 2 \\
$a$ & 1 & 1 & 1 & 1 & 2 & 2 & 2 & 2 & 2 & 2 \\
\hline$b c$ & 1 & 1 & 1 & 1 & 2 & 2 & 2 & 2 & 2 & 2 \\
$b d$ & 1 & 1 & 1 & 1 & 1 & 2 & 2 & 2 & 2 & 2 \\
$b$ & 1 & 1 & 1 & 1 & 1 & 1 & 1 & 2 & 2 & 2 \\
\hline$c d$ & 1 & 1 & 1 & 1 & 1 & 1 & 1 & 2 & 2 & 2 \\
$c$ & 1 & 1 & 1 & 1 & 1 & 1 & 1 & 1 & 1 & 2 \\
\hline$d$ & 1 & 1 & 1 & 1 & 1 & 1 & 1 & 1 & 1 & 1 \\
\hline
\end{tabular}

and such that parts with color abcd differ by at least 4 , and the smallest part with color abcd is at least equal to $4+2 \tau-\chi\left(1_{a}\right.$ is a part), where $\tau$ is the number of primary and secondary parts in the partition. The theorem is then stated as follows.

Theorem 1.5. Let $u, v, w, t, n$ be non-negative integers. Denote by $A(u, v, w, t, n)$ the number of partitions of $n$ into $u$ distncts parts with color $a, v$ distinct parts with color $b$, $w$ distinct parts with color $c$ and $t$ distinct parts with color $d$, and denote by $B(u, v, w, t, n)$ the number of partitions of $n$ satisfying the conditions in (1.11), with u parts with color a, ab, ac, ad or $a b c d, v$ parts with color $b, a b, b c, b d$ or abcd, $w$ parts with color $c, a c, b c, c d$ or abcd and $t$ parts with color $d, a d, b d, c d$ or abcd. We then have $A(u, v, w, t, n)=B(u, v, w, t, n)$ and the identity

$$
\sum_{u, v, w, t, n \geq 0} B(u, v, w, t, n) a^{u} b^{v} c^{w} d^{t} q^{n}=(-a q ; q)_{\infty}(-b q ; q)_{\infty}(-c q ; q)_{\infty}(-d q ; q)_{\infty} .
$$

Note that the result of Alladi-Andrews-Berkovich uses four primary colors, the full set of secondary colors, along with one quaternary color $a b c d$. When $d=0$, we recover Theorem 1.4. Their main tool was a difficult $q$-series identity:

$$
\begin{aligned}
\sum_{i, j, k, l-\text { constraints }} & \frac{q^{T_{\tau}+T_{A B}+T_{A C}+T_{A D}+T_{B C}+T_{B D}+T_{C D}-B C-B D-C D+4 T_{Q-1}+3 Q+2 Q \tau}}{(q)_{A}(q)_{B}(q)_{C}(q)_{D}(q)_{A B}(q)_{A C}(q)_{A D}(q)_{B C}(q)_{B D}(q)_{C D}(q)_{Q}} \\
\cdot & \left\{\left(1-q^{A}\right)+q^{A+B C+B D+Q}\left(1-q^{B}\right)+q^{A+B C+B D+Q+B+C D}\right\} \\
= & \frac{q^{T_{i}+T_{j}+T_{k}+T_{l}}}{(q)_{i}(q)_{j}(q)_{k}(q)_{l}}
\end{aligned}
$$

where $A, B, C, D, A B, A C, A D, B C, B D, C D, Q$ are variables which count the number of parts with respectively color $a, b, c, d, a b, a c, a d, b c, b d, c d, a b c d$,

$$
\left\{\begin{array}{l}
i=A+A B+A C+A D+Q \\
j=B+A B+B C+B D+Q \\
k=C+A C+B C+C D+Q \\
l=D+A D+B D+C D+Q \\
\tau=A+B+C+D+A B+A C+A D+B C+B D+C D
\end{array}\right.
$$


$T_{n}=\frac{n(n+1)}{2}$ is the $n^{\text {th }}$ triangular number and $(q)_{n}=(q ; q)_{n}$. While this identity is difficult to prove, it is relatively straightforward to show that it is equivalent to the statement in Theorem 1.5.

In this paper we give a bijective proof of Theorem 1.5 (and therefore a bijective proof of the identity (1.13) ). Our proof is divided into two steps. First we prove Theorem $\mathbf{1 . 6}$ below, which arises more naturally from our methods than Theorem 1.5. Instead of adding a quaternary color, we lower certain minimum differences and add some forbidden patterns. Then, we show how Theorem $\mathbf{1 . 6}$ is equivalent to Theorem $\mathbf{1 . 5}$.

1.2. Statement of Results. Suppose that the parts occur in only primary colors $a, b, c, d$ and secondary colors $a b, a c, a d, b c, b d, c d$, and are ordered as in (1.10) by omitting quaternary parts:

$$
1_{a b}<1_{a c}<1_{a d}<1_{a}<1_{b c}<1_{b d}<1_{b}<1_{c d}<1_{c}<1_{d}<2_{a b}<\cdots
$$

Let us now consider the partitions with the size of the secondary parts greater than one and satisfying the minimal difference conditions in

\begin{tabular}{|c|cccc|ccc|cc|c|}
\hline$\lambda_{i} \backslash^{\lambda_{i+1}}$ & $a b$ & $a c$ & $a d$ & $a$ & $b c$ & $b d$ & $b$ & $c d$ & $c$ & $d$ \\
\hline$a b$ & 2 & 2 & 2 & 2 & 2 & 2 & 2 & 2 & 2 & 2 \\
$a c$ & 1 & 2 & 2 & 2 & 2 & 2 & 2 & 2 & 2 & 2 \\
$a d$ & 1 & 1 & 2 & 2 & 1 & 2 & 2 & 2 & 2 & 2 \\
$a$ & 1 & 1 & 1 & 1 & 2 & 2 & 2 & 2 & 2 & 2 \\
\hline$b c$ & 1 & 1 & 1 & 1 & 2 & 2 & 2 & 2 & 2 & 2 \\
$b d$ & 1 & 1 & 1 & 1 & 1 & 2 & 2 & 2 & 2 & 2 \\
$b$ & 1 & 1 & 1 & 1 & 1 & 1 & 1 & 2 & 2 & 2 \\
\hline$c d$ & $\underline{0}$ & 1 & 1 & 1 & 1 & 1 & 1 & 2 & 2 & 2 \\
$c$ & 1 & 1 & 1 & 1 & 1 & 1 & 1 & 1 & 1 & 2 \\
\hline$d$ & 1 & 1 & 1 & 1 & 1 & 1 & 1 & 1 & 1 & 1 \\
\hline
\end{tabular}

and which avoid the forbidden patterns

$$
\left((k+2)_{c d},(k+2)_{a b}, k_{c}\right),\left((k+2)_{c d},(k+2)_{a b}, k_{d}\right),\left((k+2)_{a d},(k+1)_{b c}, k_{a}\right),
$$

except the pattern $\left(3_{a d}, 2_{b c}, 1_{a}\right)$ which is allowed. An example of such a partition is

$$
\left(11_{a d}, 10_{b c}, 8_{a}, 7_{c d}, 7_{a b}, 4_{c}, 3_{a d}, 2_{b c}, 1_{a}\right) \text {. }
$$

We can now state the main theorem of this paper.

Theorem 1.6. Let $u, v, w, t, n$ be non-negative integers. Denote by $A(u, v, w, t, n)$ the number of partitions of $n$ into $u$ distncts parts with color $a, v$ distinct parts with color $b, w$ distinct parts with color $c$ and $t$ distinct parts with color $d$, and denote by $B(u, v, w, t, n)$ the number of partitions of $n$ satisfying the conditions above, with $u$ parts with color a, ab, ac or ad, $v$ parts with color $b, a b, b c$ or $b d, w$ parts with color $c, a c, b c$ or $c d$ and $t$ parts with color $d, a d, b d$ or $c d$. We then have $A(u, v, w, t, n)=B(u, v, w, t, n)$, and the corresponding q-series identity is given by

$$
\sum_{u, v, w, t, n \in \mathbb{N}} B(u, v, w, t, n) a^{u} b^{v} c^{w} d^{t} q^{n}=(-a q ; q)_{\infty}(-b q ; q)_{\infty}(-c q ; q)_{\infty}(-d q ; q)_{\infty} .
$$


By specializing the variables in Theorem 1.6, one can deduce many partition identities. For example, by considering the following transformation in (1.17)

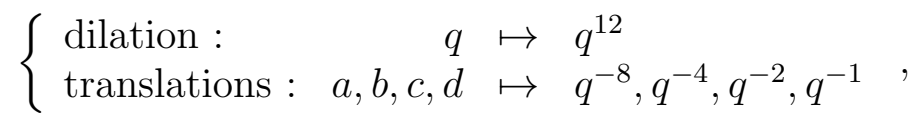

we obtain a corollary of Theorem $\mathbf{1 . 6}$.

Corollary 1.1. For any positive integer $n$, the number of partitions of $n$ into distinct parts congruent to $-2^{3},-2^{2},-2^{1},-2^{0} \bmod 12$ is equal to the number of partitions of $n$ into parts not congruent to 1,5 mod 12 and different from 2,3,6,7,9, such that the difference between two consecutive parts is greater than 12 up to the following exceptions:

- $\lambda_{i}-\lambda_{i+1}=9 \Longrightarrow \lambda_{i} \equiv \pm 3 \bmod 12$ and $\lambda_{i}-\lambda_{i+2} \geq 24$,

- $\lambda_{i}-\lambda_{i+1}=12 \Longrightarrow \lambda_{i} \equiv-2^{3},-2^{2},-2^{1},-2^{0} \bmod 12$,

except that the pattern $(27,18,4)$ is allowed.

For example, with $n=49$, the partitions of the first kind are

$$
\begin{aligned}
& (35,10,4),(34,11,4),(28,11,10),(23,22,4), \\
& (23,16,10),(22,16,11) \text { and }(16,11,10,8,4)
\end{aligned}
$$

and the partitions of the second kind are

$$
(35,14),(34,15),(33,16),(45,4),(39,10),(38,11) \text { and }(27,18,4) \text {. }
$$

Corollary 1.1 may be compared with Theorem 3 of [1], which is Theorem 1.5 transformed by (1.18) but with the dilation $q \mapsto q^{15}$ instead of $q \mapsto q^{12}$.

The paper is organized as follows. In Section 2, we will present some tools that will be useful for the proof of Theorem 1.6. After that, in Section 3, we will give the bijection for Theorem 1.6. Then, in Section 4, we will prove its well-definedness. Finally, in Section 5, we will present and prove the bijection between the partitions with forbidden patterns considered in Theorem 1.6 and the partitions with quaternary parts given in Theorem 1.5. In Part II of this series, we will show how our method can be used to go beyond Göllnitz' theorem to any number of primary colors.

\section{Preliminaries}

2.1. The setup. Denote by $\mathcal{C}=\{a, b, c, d\}$ the set of primary colors and $\mathcal{C}_{\rtimes}=\{a b, a c, a d, b c, b d, c d\}$ the set of secondary colors, and recall the order on $\mathcal{C} \sqcup \mathcal{C}_{\rtimes}$ :

$$
a b<a c<a d<a<b c<b d<b<c d<c<d .
$$

We can then define the strict lexicographic order $\succ$ on colored parts by

$$
k_{p} \succ l_{q} \Longleftrightarrow k-l \geq \chi(p \leq q) \cdot
$$

Explicitly, this gives the order

$$
1_{a b} \prec 1_{a c} \prec 1_{a d} \prec 1_{a} \prec 1_{b c} \prec 1_{b d} \prec 1_{b} \prec 1_{c d} \prec 1_{c} \prec 1_{d} \prec 2_{a b} \prec \cdots,
$$

previously etablished in (1.14). We denote by $\mathcal{P}$ the set of positive integers with primary color. 
We can easily see that for any $p q \in \mathcal{C}_{\rtimes}$, with $p<q$, and any $k \geq 1$, we have that

$$
\begin{aligned}
(2 k)_{p q} & =k_{q}+k_{p} \\
(2 k+1)_{p q} & =(k+1)_{p}+k_{q} .
\end{aligned}
$$

In fact, any part greater that 1 with a secondary color $p q$ can be uniquely written as the sum of two consecutive parts in $\mathcal{P}$ with colors $p$ and $q$. We then denote by $\mathcal{S}$ the set of secondary parts greater than 1 , and define the functions $\alpha$ and $\beta$ on $\mathcal{S}$ by

$$
\alpha:\left\{\begin{array}{lll}
2 k_{p q} & \mapsto k_{q} \\
(2 k+1)_{p q} & \mapsto(k+1)_{p}
\end{array} \quad \text { and } \quad \beta:\left\{\begin{array}{ll}
2 k_{p q} & \mapsto k_{p} \\
(2 k+1)_{p q} & \mapsto k_{q}
\end{array},\right.\right.
$$

respectively named upper and lower halves. One can check that for any $k_{p q} \in \mathcal{S}$,

$$
\alpha\left((k+1)_{p q}\right)=\beta\left(k_{p q}\right)+1 \quad \text { and } \quad \beta\left((k+1)_{p q}\right)=\alpha\left(k_{p q}\right) \cdot
$$

In the previous sum, adding an integer to a part does not change its color. We can then deduce by induction that for any $m \geq 0$,

$$
\alpha\left((k+m)_{p q}\right) \preceq \alpha\left(k_{p q}\right)+m \quad \text { and } \quad \beta\left((k+m)_{p q}\right) \preceq \beta\left(k_{p q}\right)+m \cdot
$$

Recall the table (1.11)

\begin{tabular}{|c|cccc|ccc|cc|c|}
\hline$\lambda_{i} \backslash^{\lambda_{i+1}}$ & $a b$ & $a c$ & $a d$ & $a$ & $b c$ & $b d$ & $b$ & $c d$ & $c$ & $d$ \\
\hline$a b$ & 2 & 2 & 2 & 2 & 2 & 2 & 2 & 2 & 2 & 2 \\
$a c$ & 1 & 2 & 2 & 2 & 2 & 2 & 2 & 2 & 2 & 2 \\
$a d$ & 1 & 1 & 2 & 2 & 2 & 2 & 2 & 2 & 2 & 2 \\
$a$ & 1 & 1 & 1 & 1 & 2 & 2 & 2 & 2 & 2 & 2 \\
\hline$b c$ & 1 & 1 & 1 & 1 & 2 & 2 & 2 & 2 & 2 & 2 \\
$b d$ & 1 & 1 & 1 & 1 & 1 & 2 & 2 & 2 & 2 & 2 \\
$b$ & 1 & 1 & 1 & 1 & 1 & 1 & 1 & 2 & 2 & 2 \\
\hline$c d$ & 1 & 1 & 1 & 1 & 1 & 1 & 1 & 2 & 2 & 2 \\
$c$ & 1 & 1 & 1 & 1 & 1 & 1 & 1 & 1 & 1 & 2 \\
\hline$d$ & 1 & 1 & 1 & 1 & 1 & 1 & 1 & 1 & 1 & 1 \\
\hline
\end{tabular}

It can be viewed as an order $\triangleright$ on $\mathcal{P} \sqcup \mathcal{S}$ defined by

$$
k_{p} \triangleright l_{q} \Longleftrightarrow k-l \geq 1+\left\{\begin{array}{ll}
\chi(p<q) & \text { if } \quad p \text { or } q \in \mathcal{C} \\
\chi(p \leq q) & \text { if } \quad p \text { and } q \in \mathcal{C}_{\rtimes}
\end{array} .\right.
$$

By considering the lexicographic order $\succ$, (2.9) becomes

$$
k_{p} \triangleright l_{q} \Longleftrightarrow\left\{\begin{array}{ll}
k_{p} \succeq(l+1)_{q} & \text { if } \quad p \text { or } q \in \mathcal{C} \\
k_{p} \succ(l+1)_{q} & \text { if } \quad p \text { and } q \in \mathcal{C}_{\rtimes}
\end{array} .\right.
$$

We can observe that for any primary colors $p, q$

$$
k_{p} \succ l_{q} \text { and } k_{p} \not l_{q} \Longleftrightarrow k-l=\chi(p<q) \text { and } p \neq q,
$$

and we easily check that in this case, $\left(k_{p}, l_{q}\right)=\left(\alpha\left(k_{p}+l_{q}\right), \beta\left(k_{p}+l_{q}\right)\right)$, for $k_{p}+l_{q}$ viewed as an element of $\mathcal{S}$ (see (2.4), (2.5)). 
We recall that the tables (1.15)

$\Delta=$\begin{tabular}{|c|cccc|ccc|cc|c|}
\hline$\lambda_{i} \backslash^{\lambda_{i+1}}$ & $a b$ & $a c$ & $a d$ & $a$ & $b c$ & $b d$ & $b$ & $c d$ & $c$ & $d$ \\
\hline$a b$ & 2 & 2 & 2 & 2 & 2 & 2 & 2 & 2 & 2 & 2 \\
$a c$ & 1 & 2 & 2 & 2 & 2 & 2 & 2 & 2 & 2 & 2 \\
$a d$ & 1 & 1 & 2 & 2 & 1 & 2 & 2 & 2 & 2 & 2 \\
$a$ & 1 & 1 & 1 & 1 & 2 & 2 & 2 & 2 & 2 & 2 \\
\hline$b c$ & 1 & 1 & 1 & 1 & 2 & 2 & 2 & 2 & 2 & 2 \\
$b d$ & 1 & 1 & 1 & 1 & 1 & 2 & 2 & 2 & 2 & 2 \\
$b$ & 1 & 1 & 1 & 1 & 1 & 1 & 1 & 2 & 2 & 2 \\
\hline$c d$ & 0 & 1 & 1 & 1 & 1 & 1 & 1 & 2 & 2 & 2 \\
$c$ & 1 & 1 & 1 & 1 & 1 & 1 & 1 & 1 & 1 & 2 \\
\hline$d$ & 1 & 1 & 1 & 1 & 1 & 1 & 1 & 1 & 1 & 1 \\
\hline
\end{tabular}

and (1.11) differ only when we have a pair $(p, q)$ of secondary colors such that $(p, q) \in$ $\{(c d, a b),(a d, b c)\}$. In these cases, the difference in (1.15) is one less.

We will now define a relation $\gg$ on $\mathcal{P} \sqcup \mathcal{S}$ in such a way that,

$$
k_{p} \gg l_{q} \Longleftrightarrow k-l \geq \Delta(p, q) \text {. }
$$

Using (2.10), this relation can be summarized by the following equivalence :

$$
k_{p} \gg l_{q} \Longleftrightarrow \begin{cases}k_{p} \succeq(l+1)_{q} & \text { if } p \text { or } q \in \mathcal{C} \\ k_{p} \succ(l+1)_{q} & \text { if } p \text { and } q \in \mathcal{C}_{\rtimes} \text { and }(p, q) \notin\{(c d, a b),(a d, b c)\} . \\ k_{p} \succ l_{q} & \text { if } \quad(p, q) \in\{(c d, a b),(a d, b c)\}\end{cases}
$$

We denote by $\mathcal{O}$ the set of partitions with parts in $\mathcal{P}$ and well-ordered by $\succ$. We then have that $\lambda \in \mathcal{O}$ if and only if there exist $\lambda_{1} \succ \cdots \succ \lambda_{t} \in \mathcal{P}$ such that $\lambda=\left(\lambda_{1}, \ldots, \lambda_{t}\right)$. We set $c\left(\lambda_{i}\right)$ to be the color of $\lambda_{i}$ in $\mathcal{C}$, and $C(\lambda)=c\left(\lambda_{1}\right) \cdots c\left(\lambda_{t}\right)$ as a commutative product of colors in $\mathcal{C}$. We denote by $\mathcal{E}$ the set of partitions with parts in $\mathcal{P} \sqcup \mathcal{S}$ and well-ordered by $\gg$. We then have that $\nu \in \mathcal{E}$ if and only if there exist $\nu_{1} \gg \cdots \gg \nu_{t} \in \mathcal{P} \sqcup \mathcal{S}$ such that $\nu=\left(\nu_{1}, \ldots, \nu_{t}\right)$. We set colors $c\left(\nu_{i}\right) \in \mathcal{C} \sqcup \mathcal{C}_{\rtimes}$ depending on whether $\nu_{i}$ is in $\mathcal{P}$ or $\mathcal{S}$, and we also define $C(\nu)=c\left(\nu_{1}\right) \cdots c\left(\nu_{t}\right)$ seen as a commutative product of colors in $\mathcal{C}$. In fact, a secondary color is just a product of two primary colors. For both kinds of partitions, their size is the sum of their part sizes.

We also denote by $\mathcal{E}_{1}$ the subset of partitions of $\mathcal{E}$ without the forbidden patterns,

$$
\left((k+2)_{c d},(k+2)_{a b}, k_{c}\right),\left((k+2)_{c d},(k+2)_{a b}, k_{d}\right),\left((k+2)_{a d},(k+1)_{b c}, k_{a}\right),
$$

except the pattern $\left(3_{a d}, 2_{b c}, 1_{a}\right)$ which is allowed. We finally define $\mathcal{E}_{2}$ as the subset of partitions of $\mathcal{E}$ with parts well-ordered by $\triangleright$ in (2.10), and we observe that $\mathcal{E}_{2}$ is indeed a subset of $\mathcal{E}_{1}$.

2.2. Technical lemmas. We will state and prove some important lemmas for the proof of Theorem 1.6. 
Lemma 2.1 (Ordering primary and secondary parts). For any $\left(l_{p}, k_{q}\right) \in \mathcal{P} \times \mathcal{S}$, we have the following equivalences:

$$
\begin{aligned}
& l_{p} \gg k_{q} \Longleftrightarrow(k+1)_{q} \gg(l-1)_{p}, \\
& l_{p} \gg \alpha\left(k_{q}\right) \Longleftrightarrow \beta\left((k+1)_{q}\right) \nsucc(l-1)_{p} .
\end{aligned}
$$

Lemma 2.2 (Ordering secondary parts). Let us consider the table $\Delta$ in (1.15). Then, for any secondary colors $p, q \in \mathcal{C}_{\rtimes}$,

$$
\Delta(p, q)=\min \left\{k-l: \beta\left(k_{p}\right) \succ \alpha\left(l_{q}\right)\right\} .
$$

Moreover, if the secondary parts $k_{p}, l_{q}$ are such that $\beta\left(k_{p}\right) \succ \beta\left(l_{q}\right)$, then

$$
(k+1)_{p} \gg l_{q} \text {. }
$$

Furthermore, if $k_{p} \gg l_{q}$, we then have either $\beta\left(k_{p}\right) \succ \alpha\left(l_{q}\right)$ or

$$
\alpha\left(l_{q}\right)+1 \gg \alpha\left((k-1)_{p}\right) \succ \beta\left((k-1)_{p}\right) \succ \beta\left(l_{q}\right) \cdot
$$

Lemma 2.3 (Reversibility $\left.\mathcal{O} \leftarrow \mathcal{E}_{1}\right)$. Let us consider a partition $\nu=\left(\nu_{1}, \ldots, \nu_{t}\right) \in$ $\mathcal{E}$. Then, for any $i \in[1, t-2]$ such that $\left(\nu_{i+1}, \nu_{i+2}\right) \in \mathcal{S} \times \mathcal{P}$ and $\left(c\left(\nu_{i}\right), c\left(\nu_{i+1}\right)\right) \notin$ $\{(a d, b c),(c d, a b)\}$, we have

$$
\nu_{i} \succ \nu_{i+2}+2
$$

Furthermore, the following are equivalent:

(1) $\nu \in \mathcal{E}_{1}$,

(2) For any $i \in[1, t-2]$ such that $\left(\nu_{i}, \nu_{i+1}\right)$ is a pattern in $\left\{\left((k+1)_{a d}, k_{b c}\right),\left(k_{c d}, k_{a b}\right)\right\}$ different from $\left(3_{a d}, 2_{b c}\right)$, we have that

$$
\nu_{i} \succeq \nu_{i+2}+2 \text {. }
$$

Proof of Lemma [2.1. To prove (2.15), we observe that, for any $\left(l_{p}, k_{q}\right) \in \mathcal{P} \times \mathcal{S}$, by (2.13),

$$
l_{p} \ngtr k_{q} \Longleftrightarrow l_{p} \nsucceq(k+1)_{q},
$$

and

$$
\begin{aligned}
(k+1)_{q} \gg(l-1)_{p} & \Longleftrightarrow(k+1)_{q} \succ l_{p} \\
& \Longleftrightarrow(k+1)_{q} \npreceq l_{p} .
\end{aligned}
$$

To prove (2.16), we first remark that, by (2.7), $\alpha\left(k_{q}\right)=\beta\left((k+1)_{q}\right)$. We then obtain by (2.13) that

$$
l_{p} \gg \alpha\left(k_{q}\right) \Longleftrightarrow(l-1)_{p} \succeq \alpha\left(k_{q}\right)
$$

and

$$
\begin{aligned}
\beta\left((k+1)_{q}\right) \nsucc(l-1)_{p} & \Longleftrightarrow \alpha\left(k_{q}\right) \nsucc(l-1)_{p} \\
& \Longleftrightarrow \alpha\left(k_{q}\right) \preceq(l-1)_{p} .
\end{aligned}
$$

Proof of Lemma 2.2. Let us consider $\min \left\{k-l: \beta\left(k_{p}\right) \succ \alpha\left(l_{q}\right)\right\}$. We just check for the 36 pairs $(p, q)$ in $\mathcal{C}_{\rtimes}^{2}$. As an example, we take the pairs $(c d, a b),(a d, b c)$. 
- For $k=2 k^{\prime}+1$, we have $\left(\alpha\left(k_{c d}\right), \beta\left(k_{c d}\right)\right)=\left(\left(k^{\prime}+1\right)_{c}, k_{d}^{\prime}\right)$. Then, to minimize $k-l$, $\alpha\left(l_{a b}\right)$ and $\beta\left(l_{a b}\right)$ have to be the greatest primary parts with color $a, b$ less than $k_{d}^{\prime}$. So we obtain $k_{b}^{\prime}$ and $k_{a}^{\prime}$. We then have

$$
k-l=2 k^{\prime}+1-2 k^{\prime}=1 .
$$

For $k=2 k^{\prime}$, we have $\left(\alpha\left(k_{c d}\right), \beta\left(k_{c d}\right)\right)=\left(k_{d}^{\prime}, k_{c}^{\prime}\right)$. Then to minimize $k-l, \alpha\left(l_{a b}\right)$ and $\beta\left(l_{a b}\right)$ have to be the greatest primary parts with color $a, b$ less than $k_{c}^{\prime}$. So we obtain $k_{b}^{\prime}$ and $k_{a}^{\prime}$. We then have

$$
k-l=2 k^{\prime}-2 k^{\prime}=0 .
$$

Therefore, $\Delta(c d, a b)=0$.

- We check with the same reasoning by taking for $(a d, b c)$ consecutive parts

$$
(k+1)_{a} \succ k_{d} \succ k_{c} \succ k_{b}
$$

and

$$
(k+1)_{d} \succ(k+1)_{a} \succ k_{c} \succ k_{b},
$$

and we obtain $\Delta(a d, b c)=1$.

To prove (2.18), we have by (2.7) that $\alpha\left((l-1)_{q}\right)=\beta\left(l_{q}\right)$. Since $\beta\left(k_{p}\right) \succ \beta\left(l_{q}\right)=\alpha\left((l-1)_{q}\right)$, this then implies by (2.17) that $k_{p} \gg(l-1)_{q}$, and this is equivalent to $(k+1)_{p} \gg l_{q}$.

Let us now suppose that $k-l \geq \Delta(p, q)$. We just saw that this minimum value was reached at $k$ or $k-1$. Then if we do not have $\beta\left(k_{p}\right) \succ \alpha\left(l_{q}\right)$, we necessarily have $\beta\left((k-1)_{p}\right) \succ$ $\alpha\left((l-1)_{q}\right)=\beta\left(l_{q}\right)$ by (2.7). Moreover, by (2.13), we have

$$
\beta\left(k_{p}\right) \nsucc \alpha\left(l_{q}\right) \Longleftrightarrow \alpha\left(l_{q}\right)+1 \gg \alpha\left((k-1)_{p}\right),
$$

so that we obtain (2.19).

Proof of Lemma [2.3. For any $\nu=\left(\nu_{1}, \ldots, \nu_{t}\right) \in \mathcal{E}$ and any $i \in[1, t-2]$, we have $\nu_{i} \gg$ $\nu_{i+1} \gg \nu_{i+2}$. By (2.13), the fact that $\left(\nu_{i+1}, \nu_{i+2}\right) \in \mathcal{S} \times \mathcal{P}$ implies that

$$
\nu_{i+1} \succ \nu_{i+2}+1
$$

and $\left(c\left(\nu_{i}\right), c\left(\nu_{i+1}\right)\right) \notin\{(a d, b c),(c d, a b)\}$ implies that

$$
\nu_{i} \succ \nu_{i+1}+1
$$

and we thus have (2.20).

To prove the second part, we have to show that not having the forbidden patterns in (2.14) is equivalent to the second condition.

- If we suppose that $\left(\nu_{i}, \nu_{i+1}\right)=\left(k_{c d}, k_{a b}\right)$, we then have by (2.13) that

$$
\begin{aligned}
\nu_{i+1} \gg \nu_{i+2} & \Longleftrightarrow \nu_{i+1} \succ \nu_{i+2}+1 \\
& \Longleftrightarrow k_{a b} \succ \nu_{i+2}+1 \\
& \Longleftrightarrow(k-1)_{d} \succeq \nu_{i+2}+1 \quad(\text { by }(2.3)) .
\end{aligned}
$$


By (2.3), we then have that the fact that the patterns $k_{c d}, k_{a b},(k-2)_{d}$ and $k_{c d}, k_{a b},(k-$ $2)_{c}$ are forbidden for $k \geq 3$ is equivalent to $(k-1)_{c d} \succeq \nu_{i+2}+1$, which means that $k_{c d} \succeq \nu_{i+2}+2$.

- If we suppose that $\left(\nu_{i}, \nu_{i+1}\right)=\left((k+1)_{a d}, k_{b c}\right)$ with $k \geq 3$, we then have by (2.13) that

$$
\begin{aligned}
\nu_{i+1} \gg \nu_{i+2} & \Longleftrightarrow \nu_{i+1} \succ \nu_{i+2}+1 \\
& \Longleftrightarrow k_{c b} \succ \nu_{i+2}+1 \\
& \Longleftrightarrow k_{a} \succeq \nu_{i+2}+1 \quad(\text { by }(\underline{2.3})) .
\end{aligned}
$$

We then have by (2.3) that the fact that the pattern $(k+1)_{a d}, k_{b c},(k-1)_{a}$ is forbidden for $k \geq 3$ is equivalent to $k_{a d} \succeq \nu_{i+2}+1$, which means that $(k+1)_{a d} \succeq \nu_{i+2}+2$.

\section{BREssoud's AlgORITHM}

Here we adapt the algorithm given by Bressoud in his bijective proof of Schur's partition theorem [6]. The bijection is easy to describe and execute, but its justification is more subtle and is given in the next section.

3.1. From $\mathcal{O}$ to $\mathcal{E}_{1}$. Let us consider the following machine $\Phi$ :

Step 1: For a sequence $\lambda=\lambda_{1}, \ldots, \lambda_{t}$, take the smallest $i<t$ such that $\lambda_{i}, \lambda_{i+1} \in \mathcal{P}$ and $\lambda_{i} \succ \lambda_{i+1}$ but $\lambda_{i} \gg \lambda_{i+1}$, if it exists, and replace

$$
\begin{array}{ll}
\lambda_{i} \longleftarrow \lambda_{i}+\lambda_{i+1} & \text { as a part in } \mathcal{S} \\
\lambda_{j} \leftarrow \lambda_{j+1} & \text { for all } i<j<t
\end{array}
$$

and move to Step 2. We call such a pair of parts a troublesome pair. We observe that $\lambda$ loses two parts in $\mathcal{P}$ and gains one part in $\mathcal{S}$. The new sequence is $\lambda=\lambda_{1}, \ldots, \lambda_{t-1}$. Otherwise, exit from the machine.

Step 2: For $\lambda=\lambda_{1}, \ldots, \lambda_{t}$, take the smallest $i<t$ such that $\left(\lambda_{i}, \lambda_{i+1}\right) \in \mathcal{P} \times \mathcal{S}$ and $\lambda_{i} \ngtr \lambda_{i+1}$ if it exists, and replace

$$
\left(\lambda_{i}, \lambda_{i+1}\right) \hookrightarrow\left(\lambda_{i+1}+1, \lambda_{i}-1\right) \in \mathcal{S} \times \mathcal{P}
$$

and redo Step 2. We say that the parts $\lambda_{i}, \lambda_{i+1}$ are crossed. Otherwise, move to Step 1.

Let $\Phi(\lambda)$ be the resulting sequence after putting any $\lambda=\left(\lambda_{1}, \ldots, \lambda_{t}\right) \in \mathcal{O}$ in $\Phi$. This transformation preserves the size and the commutative product of primary colors of partitions. 
Let us apply this machine on the partition $\left(11_{c}, 8_{d}, 6_{a}, 4_{d}, 4_{c}, 4_{b}, 3_{a}, 2_{b}, 2_{a}, 1_{d}, 1_{c}, 1_{b}, 1_{a}\right)$.

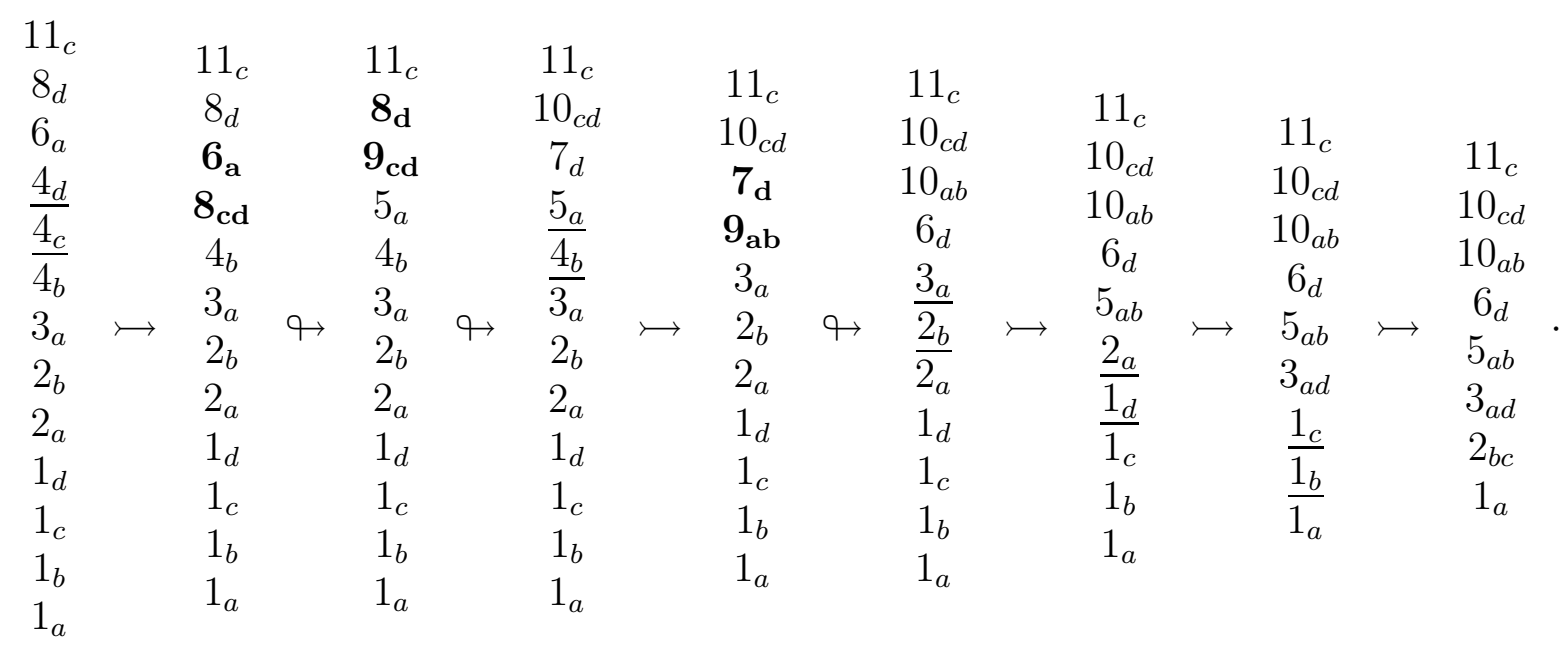

3.2. From $\mathcal{E}_{1}$ to $\mathcal{O}$. Let us consider the following machine $\Psi$ :

Step 1: For a sequence $\nu=\nu_{1}, \ldots, \nu_{t}$, take the greastest $i \leq t$ such that $\nu_{i} \in \mathcal{S}$ if it exists. If $\nu_{i+1} \in \mathcal{P}$ and $\beta\left(\nu_{i}\right) \nsucc \nu_{i+1}$, then replace

$$
\left(\nu_{i}, \nu_{i+1}\right) \uparrow\left(\nu_{i+1}+1, \nu_{i}-1\right) \in \mathcal{P} \times \mathcal{S}
$$

and redo Step 1. We say that the parts $\nu_{i}, \nu_{i+1}$ are crossed. Otherwise, move to Step 2. If there are no more parts in $\mathcal{S}$, exit from the machine.

Step 2: For $\nu=\nu_{1}, \ldots, \nu_{t}$, take the the greatest $i \leq t$ such that $\nu_{i} \in \mathcal{S}$. By Step 1, it satisfies $\beta\left(\nu_{i}\right) \succ \nu_{i+1}$. Then replace

$$
\begin{array}{ll}
\nu_{j+1} \leftrightarrows \nu_{j} & \text { for all } t \geq j>i \\
\left(\nu_{i}\right) \rightrightarrows\left(\alpha\left(\nu_{i}\right), \beta\left(\nu_{i}\right)\right) & \text { as a pair of parts in } \mathcal{P},
\end{array}
$$

and move to Step 1. We say that the part $\nu_{i}$ splits. We observe that $\nu$ gains two parts in $\mathcal{P}$ and loses one part in $\mathcal{S}$. The new sequence is $\nu=\nu_{1}, \ldots, \nu_{t+1}$.

Let $\Psi(\nu)$ be the resulting sequence after putting any $\nu=\left(\nu_{1}, \ldots, \nu_{t}\right) \in \mathcal{E}_{1}$ in $\Psi$. This transformation preserves the size and the product of primary colors of partitions. For example, applying this to $\left(11_{c}, 10_{c d}, 10_{a b}, 6_{d}, 5_{a b}, 3_{a d}, 2_{b c}, 1_{a}\right)$ gives

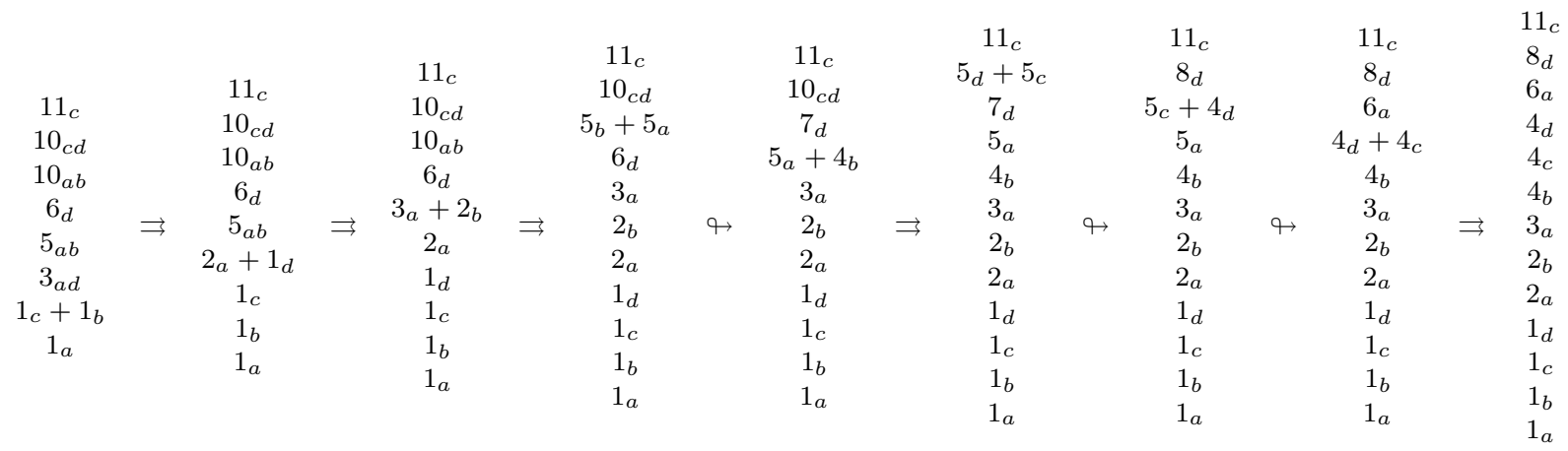




\section{Proof of the Well-Definedness of Bressoud's MAPS}

In this section, we will show the following proposition.

Proposition 4.1. The transformation $\Phi$ describes a mapping from $\mathcal{O}$ to $\mathcal{E}_{1}$ such that $\Psi \circ \Phi=$ $I d_{\mathcal{O}}$, and $\Psi$ describes a mapping from $\mathcal{E}_{1}$ to $\mathcal{O}$ such that $\Phi \circ \Psi=I d_{\mathcal{E}_{1}}$.

4.1. Well-definedness of $\Phi$. In this subsection, we will show the following proposition.

Proposition 4.2. Let us consider any $\lambda=\left(\lambda_{1}, \ldots, \lambda_{t}\right) \in \mathcal{O}$, and set $\gamma^{0}=0, \mu^{0}=\lambda$. Then, in the process $\Phi$ on $\lambda$, at the $u^{\text {th }}$ passage from Step 2 to Step $\mathbf{1}$, there exists a pair of partitions $\gamma^{u}, \mu^{u} \in \mathcal{E}_{1} \times \mathcal{O}$ such that the sequence obtained is $\gamma^{u}, \mu^{u}$. Moreover, if we denote by $l\left(\gamma^{u}\right)$ and $g\left(\mu^{u}\right)$ respectively the smallest part of $\gamma^{u}$ and the greatest part of $\mu^{u}$, we then have that

(1) $l\left(\gamma^{u}\right)$ is the $u^{\text {th }}$ element in $\mathcal{S}$ of $\gamma^{u}$,

(2) $l\left(\gamma^{u}\right) \gg g\left(\mu^{u}\right)$ so that the partition $\left(\gamma^{u}, g\left(\mu^{u}\right)\right)$ is in $\mathcal{E}_{1}$,

(3) for any $u, \gamma^{u}$ is the beginning of the partition $\gamma^{u+1}$ and the number of parts of $\mu^{u+1}$ is at least two less than the number of parts of $\mu^{u}$.

Proof. Let $\lambda=\left(\lambda_{1}, \ldots, \lambda_{t}\right)$ be a partition in $\mathcal{O}$. Let us set $c_{1}, \ldots, c_{t}$ to be the primary colors of the parts $\lambda_{1}, \ldots, \lambda_{t}$. Now consider the first troublesome pair $\lambda_{i}, \lambda_{i+1} \in \mathcal{P}$ obtained at Step 1 in $\Phi$, and the first resulting secondary part $\lambda_{i}+\lambda_{i+1}$. Note that this is reversible by Step 2 of $\Psi$.

- If there is a part $\lambda_{i+2}$ after $\lambda_{i+1}$, we have that

$$
\begin{aligned}
\lambda_{i}+\lambda_{i+1}-\lambda_{i+2} & =\chi\left(c_{i}<c_{i+1}\right)+2 \lambda_{i+1}-\lambda_{i+2} \quad \text { by (2.11) } \\
& \geq \chi\left(c_{i}<c_{i+1}\right)+2 \chi\left(c_{i+1} \leq c_{i+2}\right)+\lambda_{i+2} \quad \text { by (2.2) } \\
& \geq 1+\chi\left(c_{i} \leq c_{i+2}\right)+\chi\left(c_{i+1} \leq c_{i+2}\right) .
\end{aligned}
$$

Since by (2.1), we have that $c_{i}>c_{i+2}$ and $c_{i+1}>c_{i+2}$ implies $c_{i} c_{i+1}>c_{i+2}$, we then have that $\lambda_{i}+\lambda_{i+1}-\lambda_{i+2} \geq 1+\chi\left(c_{i} c_{i+1} \leq c_{i+2}\right)$, and we conclude that $\lambda_{i}+\lambda_{i+1} \gg \lambda_{i+2}$.

- The primary parts to the left of $\lambda_{i}$ are well-ordered by $\gg$. We then have

$$
\lambda_{1} \gg \cdots \gg \lambda_{i-1} \gg \lambda_{i}
$$

We obtain by (2.13) and (2.8) that for any $j<i$,

$$
\lambda_{j} \gg \lambda_{i}+i-j-1 \succeq \alpha\left(\lambda_{i}+\lambda_{i+1}+i-j-1\right)
$$

so that by (2.13),$\lambda_{j} \gg \alpha\left(\lambda_{i}+\lambda_{i+1}+i-j-1\right)$. If after $i-j$ iterations of Step $\mathbf{2}$, $\lambda_{i}+\lambda_{i+1}$ crosses $\lambda_{j}$, we will have at the same time that

$$
\begin{gathered}
\left(\lambda_{i}+\lambda_{i+1}+i-j\right) \gg\left(\lambda_{j}-1\right) \gg \cdots \gg \lambda_{i-1}-1 \quad(\text { by }(\underline{2.15})) \\
\beta\left(\lambda_{i}+\lambda_{i+1}+i-j\right) \nsucc\left(\lambda_{j}-1\right) \gg \cdots \gg \lambda_{i-1}-1 \quad(\text { by }(2.16)),
\end{gathered}
$$

and so these iterations are reversible by Step 1 in $\Psi$ (recursively on $j \leq j^{\prime}<i$ ).

- We also have by (2.13) that

$$
\begin{aligned}
\lambda_{i-1} \gg \lambda_{i} \succ \lambda_{i+1} \succ \lambda_{i+2} & \Longrightarrow \lambda_{i-1}-1 \succeq \lambda_{i} \succ \lambda_{i+1} \succ \lambda_{i+2} \\
& \Longrightarrow \lambda_{i-1}-1 \succ \lambda_{i+2} .
\end{aligned}
$$


- If we can no longer apply Step 2 after $i-j$ iterations, we then obtain $\lambda_{1} \gg \cdots \gg \lambda_{j-1} \gg\left(\lambda_{i}+\lambda_{i+1}+i-j\right) \gg\left(\lambda_{j}-1\right) \gg \cdots \gg \lambda_{i-1}-1 \succ \lambda_{i+2} \succ \cdots \succ \lambda_{t}$ and we set

$$
\begin{aligned}
& \gamma^{1}=\lambda_{1} \gg \cdots \gg\left(\lambda_{i}+\lambda_{i+1}+i-j\right) \\
& \mu^{1}=\left(\lambda_{j}-1\right) \gg \cdots \gg \lambda_{i-1}-1 \succ \lambda_{i+2} \succ \cdots \succ \lambda_{t},
\end{aligned}
$$

and the conditions in the proposition are respected. In fact, even if $j=i$, we saw that $\lambda_{i}+\lambda_{i+1} \gg \lambda_{i+2}$.

Now, by applying Step 1 for the second time, we see by (4.4) that the next troublesome pair is either $\lambda_{i-1}-1, \lambda_{i+2}$, or $\lambda_{i+2+x}, \lambda_{i+3+x}$ for some $x \geq 0$.

- If $\lambda_{i-1}-1 \ngtr \lambda_{i+2}$, this means that $\lambda_{i-1}-1, \lambda_{i+2}$ are consecutive for $\succ$, and Step 1 occurs there. By (2.18), we have that $\left(\lambda_{i}+\lambda_{i+1}+1\right) \gg\left(\lambda_{i-1}+\lambda_{i+2}-1\right)$. Then, even if $\left(\lambda_{i-1}+\lambda_{i+2}-1\right)$ crosses the primary parts $\left(\lambda_{j}-1\right) \gg \cdots \gg \lambda_{i-2}-1$ after $i-j-1$ iterations of Step 2, by (2.13), we will still have that

$$
\left(\lambda_{i}+\lambda_{i+1}+i-j\right) \gg\left(\lambda_{i-1}+\lambda_{i+2}+i-j-2\right) \cdot
$$

- If $\lambda_{i-1}-1 \gg \lambda_{i+2}$, then the next troublesome pair appears at $\lambda_{i+2+x}, \lambda_{i+3+x}$ for some $x \geq 0$, and it forms the secondary part $\lambda_{i+2+x}+\lambda_{i+3+x}$. We also have

$$
\lambda_{i} \succ \lambda_{i+1} \succ \lambda_{i+2} \gg \cdots \gg \lambda_{i+2+x} \succ \lambda_{i+3+x} .
$$

By (2.13), we can easily check that

$$
\lambda_{i} \succ \lambda_{i+1} \succ \lambda_{i+2} \succeq \lambda_{i+2+x}+x \succ \lambda_{i+3+x}+x
$$

so that, by (2.17),

$$
\left(\lambda_{i}+\lambda_{i+1}\right) \gg\left(\lambda_{i+2+x}+\lambda_{i+3+x}+2 x\right) .
$$

This means by (2.13) that,

$$
\left(\lambda_{i}+\lambda_{i+1}\right) \gg\left(\lambda_{i+2+x}+\lambda_{i+3+x}+x\right)
$$

and, as soon as $x \geq 1$, by (2.9)

$$
\left(\lambda_{i}+\lambda_{i+1}\right) \triangleright\left(\lambda_{i+2+x}+\lambda_{i+3+x}+x\right) \cdot
$$

We then obtain that, even if the secondary part $\lambda_{i+2+x}+\lambda_{i+3+x}$ crosses, after $x+i-j$ iterations of Step 2, the primary parts

$$
\lambda_{j}-1 \gg \cdots \gg\left(\lambda_{i-1}-1\right) \gg \lambda_{i+2} \gg \cdots \gg \lambda_{i+1+x},
$$

we will still have

$$
\left(\lambda_{i}+\lambda_{i+1}+i-j\right) \gg\left(\lambda_{i+2+x}+\lambda_{i+3+x}+x+i-j\right) \cdot
$$

However, as soon as $x \geq 1$, we directly have

$$
\left(\lambda_{i}+\lambda_{i+1}+i-j\right) \triangleright\left(\lambda_{i+2+x}+\lambda_{i+3+x}+x+i-j\right) \cdot
$$

In that case, the pair $\left(\lambda_{i}+\lambda_{i+1}+i-j, \lambda_{i+2+x}+\lambda_{i+3+x}+x+i-j\right)$ cannot have the form $\left(k_{c d}, k_{a b}\right)$ or $\left((k+1)_{a d}, k_{b c}\right)$. In order to have these patterns, we must necessarily have that the second troublesome pair is either $\left(\lambda_{i-1}-1, \lambda_{i+2}\right)$ or $\left(\lambda_{i+2}, \lambda_{i+3}\right)$. In both cases, we can 
see that either both parts crossed the primary part $l_{s}$ to the right of the pattern, or they do not move backward, so that the lower half of the second secondary part is greater than the primary part $l_{s}$ to the right of the pattern in terms of $\succ$. In the first case, we have that

$$
\begin{aligned}
& (l+2)_{s} \not \lambda_{i}+\lambda_{i+1}+i-j-1 \\
\Longleftrightarrow \quad & (l+2)_{s} \nsucceq \lambda_{i}+\lambda_{i+1}+i-j \quad \text { by (2.13) }
\end{aligned}
$$

and then $\lambda_{i}+\lambda_{i+1}+i-j \succ(l+2)_{s}$, so that the forbidden patterns in (2.14) do not occur. In the second case, we check the different subcases:

$$
\begin{aligned}
\left(2 k_{c d}^{\prime}, 2 k_{a b}^{\prime}, l_{s}\right) & \Longrightarrow k_{a}^{\prime} \succ l_{s} \\
& \Longrightarrow k^{\prime}-l \geq \chi(a \leq s)=1 \\
& \Longrightarrow 2 k^{\prime}-l \geq l+2 \geq 3 \\
& \Longrightarrow 2 k^{\prime}-(l+2) \geq 1 \geq \chi(c d \leq s), \\
\left(\left(2 k^{\prime}+1\right)_{c d},\left(2 k^{\prime}+1\right)_{a b}, l_{s}\right) \Longrightarrow & k_{b}^{\prime} \succ l_{s} \\
\Longrightarrow & k^{\prime}-l \geq \chi(b \leq s) \\
\Longrightarrow & 2 k^{\prime}+1-l \geq l+1+2 \chi(b \leq s) \\
\Longrightarrow & 2 k^{\prime}+1-l \geq 2+2 \chi(b \leq s) \\
\Longrightarrow & \left(2 k^{\prime}+1\right)-(l+2) \geq \chi(b \leq s) \geq \chi(c d \leq s) \quad \text { since } \quad b<c d . \\
\left(\left(2 k^{\prime}+2\right)_{a d},\left(2 k^{\prime}+1\right)_{b c}, l_{s}\right) & \Longrightarrow k_{c}^{\prime} \succ l_{s} \\
& \Longrightarrow k^{\prime}-l \geq \chi(c \leq s) \\
& \Longrightarrow 2 k^{\prime}-l \geq l+2 \chi(c \leq s) \\
& \Longrightarrow 2 k^{\prime}-l \geq 1+2 \chi(c \leq s) \\
& \Longrightarrow\left(2 k^{\prime}+2\right)-(l+2) \geq 1+\chi(c \leq s) \geq \chi(a d \leq s) \cdot \\
\Longrightarrow &
\end{aligned}
$$

We can see that only $\left(3_{a d}, 2_{b c}, 1_{a}\right)$ does not satisfy the fact that $(l+2)_{s}$ is less than the first part $\lambda_{i}+\lambda_{i+1}+i-j$. Recall that the second part needs to be greater than $(l+1)_{s}$. By Lemma 2.3, we then have the forbidden patterns

$$
\left((k+2)_{c d},(k+2)_{a b}, k_{c}\right),\left((k+2)_{c d},(k+2)_{a b}, k_{d}\right),\left((k+2)_{a d},(k+1)_{b c}, k_{a}\right),
$$

with only $\left(3_{a d}, 2_{b c}, 1_{a}\right)$ allowed. The conditions in the proposition are satisfied after the second move from Step 2 to Step 1.

By induction, Proposition 4.2 follows. Moreover, by (4.2), every single step is reversible 
by $\Psi$, since by its application the sequence $\gamma^{u+1}, \mu^{u+1}$ becomes exactly after the iterations of Step 1 and splitting in Step 2 the sequence $\gamma^{u}, \mu^{u}$ (with the last part of $\gamma^{u+1}$ ).

The fact that $\Phi(\mathcal{O}) \subset \mathcal{E}_{1}$ follows from Proposition 4.2 since $\mu^{u}$ strictly decreases in terms of number of parts and the process stops as soon as either $\mu^{u}$ has at most one part, or all its primary parts are well-ordered by $\gg$. And the reversibility implies that $\Psi \circ \Phi_{\mid \mathcal{O}}=I d_{\mathcal{O}}$.

4.2. Well-definedness of $\Psi$. In this subsection, we will show the following proposition.

Proposition 4.3. Let us consider any $\nu=\nu_{1}, \ldots, \nu_{t} \in \mathcal{E}_{1}$, and set $\gamma^{0}=\nu, \mu^{0}=0$. Then, in the process $\Psi$ on $\nu$, at the $u^{\text {th }}$ passage from Step $\mathcal{2}$ to Step 1 , there exists a pair of partitions $\gamma^{u}, \mu^{u} \in \mathcal{E}_{1} \times \mathcal{O}$ such that the sequence obtained is $\gamma^{u}, \mu^{u}$. Moreover, if we denote by $l\left(\gamma^{u}\right)$ and $g\left(\mu^{u}\right)$ respectively the smallest part of $\gamma^{u}$ and the greatest part of $\mu^{u}$, we then have that

(1) $l\left(\gamma^{u}\right) \in \mathcal{P}$,

(2) $l\left(\gamma^{u}\right)$ and $g\left(\mu^{u}\right)$ are consecutive for $\succ$,

(3) for any $u, \mu^{u}$ is the tail of the partition $\mu^{u+1}$ and the number of secondary parts of $\gamma^{u}$ decreases by one at each step.

Proof. If the pattern $\left(3_{a d}, 2_{b c}, 1_{a}\right)$ is in $\nu$, these parts are then the last ones. By applying $\Psi$, we obtain after the second passage at the tail of the partition the sequence $2_{a}, 1_{d}, 1_{c}, 1_{b}, 1_{a}$. Now suppose that this pattern does not occur in $\nu$. Let us consider the last secondary part $\nu_{i}$ of $\nu$.

- Suppose that Step 1 does not occur and we directly have Step 2. If there is a part $\nu_{i-1}$ to its left, and $\left(\nu_{i-1}, \nu_{i}\right) \notin\left\{\left((k+1)_{a d}, k_{b c}\right),\left(k_{c d}, k_{a b}\right)\right\}$, we then have $\nu_{i-1} \triangleright \nu_{i}$ and

$$
\begin{aligned}
\nu_{i-1}-\alpha\left(\nu_{i}\right) & =\nu_{i-1}-\nu_{i}+\beta\left(\nu_{i}\right) \\
& \left.\geq 2 \quad(\text { by (2.9) }) \text { and the fact that } \beta\left(\nu_{i}\right) \geq 1\right),
\end{aligned}
$$

so that $\nu_{i-1} \gg \alpha\left(\nu_{i}\right)$. In the case that $\left(\nu_{i-1}, \nu_{i}\right) \in\left\{\left((k+1)_{a d}, k_{b c}\right),\left(k_{c d}, k_{a b}\right)\right\}$, a quick check according to the parity of $k$ shows that we also have $\nu_{i-1} \gg \alpha\left(\nu_{i}\right)$. If we have the pattern $\left(\nu_{i-2}, \nu_{i-1}\right) \in\left\{\left((k+1)_{a d}, k_{b c}\right),\left(k_{c d}, k_{a b}\right)\right\}$, then $\nu_{i} \preceq \nu_{i-2}-2$, and

$$
\begin{aligned}
\nu_{i-2}-\alpha\left(\nu_{i}\right) & =\nu_{i-2}-\nu_{i}+\beta\left(\nu_{i}\right) \\
& \geq 3 \quad \text { by }(\underline{2.9}) \text { and the fact that } \beta\left(\nu_{i}\right) \geq 1
\end{aligned}
$$

so that $\nu_{i-2} \succ\left(\alpha\left(\nu_{i}\right)+2\right)$. Note that by Lemma 2.3, this implies that $\nu_{i-2}, \nu_{i-1}, \alpha\left(\nu_{i}\right)$ cannot be a forbidden pattern.

- If $\nu_{i}$ crosses after iteration of Step 1 the primary parts $\nu_{i+1} \gg \cdots \gg \nu_{j}$, we then have

$$
\begin{aligned}
& \nu_{i-1} \gg \nu_{i+1}+1 \gg \cdots \gg \nu_{j}+1 \gg \alpha\left(\nu_{i}-j+i\right) \quad(\text { by (2.16) }) \\
& \nu_{i-1} \gg \nu_{i+1}+1 \gg \cdots \gg \nu_{j}+1 \gg\left(\nu_{i}-j+i\right) .
\end{aligned}
$$

In fact, by (2.20) $)$, if $\left(\nu_{i-1}, \nu_{i}\right) \notin\left\{\left((k+1)_{a d}, k_{b c}\right),\left(k_{c d}, k_{a b}\right)\right\}$, we necessarily have that $\nu_{i-1} \gg \nu_{i} \gg \nu_{i+1}$ so that $\nu_{i-1} \gg \nu_{i+1}+1$. If $\left(\nu_{i-1}, \nu_{i}\right) \in\left\{\left((k+1)_{a d}, k_{b c}\right),\left(k_{c d}, k_{a b}\right)\right\}$, since $\nu_{i-1} \succ\left(\nu_{i+1}+2\right) \in \mathcal{P}$, we necessarily have by (2.13) that $\nu_{i-1} \gg\left(\nu_{i+1}+1\right)$. 
If we have the pattern $\left(\nu_{i-2}, \nu_{i-1}\right) \in\left\{\left((k+1)_{a d}, k_{b c}\right),\left(k_{c d}, k_{a b}\right)\right\}$, then $\nu_{i} \preceq \nu_{i-2}-2$, and

$$
\nu_{i-2} \succeq \nu_{i}+2 \succ \nu_{i+1}+3
$$

So $\nu_{i-2} \succ \nu_{i+1}+3$, and the pattern $\nu_{i-2}, \nu_{i-1}, \nu_{i+1}+1$ is not forbidden.

Finally, since $\nu_{i} \gg \nu_{i+1} \gg \cdots \gg \nu_{j}$ and $\nu_{i+1}, \ldots, \nu_{j} \in \mathcal{P}$, we then have by (2.13) that $\nu_{i} \gg \nu_{j}+j-i-1$, and this is equivalent by (2.15) to $\nu_{j}+1 \gg\left(\nu_{i}-j+i\right)$. This implies that all these iterations of Step 1 are reversible by Step 2 of $\Phi$.

- In the case $j=t$, we have by (4.9), (2.15) and (2.13) that

$$
\nu_{i}-t+i \succ \nu_{t}
$$

If we suppose that $\nu_{i}-t+i$ has size 1 , then $\nu_{t}$ has also size 1 and a color smaller than the color of $\nu_{i}$. But by (2.4) and (2.1), we necessarily have that $\beta\left(\nu_{i}-t+i+1\right)$ has size 1 and a color greater than the color of $\nu_{i}$. We then obtain by (2.3) that

$$
\beta\left(\nu_{i}-t+i+1\right) \succ \nu_{i}-t+i \succ \nu_{t},
$$

so that we do not cross $\nu_{i}-t+i+1$ and $\nu_{t}$. This is absurd by assumption. In any case, after crossing, we still have that the secondary part size is greater than 1 , so that after splitting, its upper and lower halves stay in $\mathcal{P}$.

- If we stop the iteration of Step 1 just before $\nu_{j+1}$, this means by (4.8) that (4.10) $\nu_{i-1} \gg \nu_{i+1}+1 \gg \cdots \gg \nu_{j}+1 \gg \alpha\left(\nu_{i}-j+i\right) \succ \beta\left(\nu_{i}-j+i\right) \succ \nu_{j+1} \gg \cdots \gg \nu_{t}$.

We then set

$$
\begin{aligned}
& \gamma^{1}=\nu_{1} \gg \cdots \gg \nu_{i-1} \gg \nu_{i+1}+1 \gg \cdots \gg \nu_{j}+1 \gg \alpha\left(\nu_{i}-j+i\right), \\
& \mu^{1}=\beta\left(\nu_{i}-j+i\right) \succ \nu_{j+1} \succ \cdots \succ \nu_{t},
\end{aligned}
$$

and we saw with all the different cases that the conditions of Proposition 4.3 are respected.

Let us now consider the secondary part $\nu_{i-x}$ before $\nu_{i}$, for some $x \geq 1$. Then, by iteration of Step 1, it can never cross $\beta\left(\nu_{i}-j+i\right)$. In fact, suppose that it crosses all primary parts $\nu_{i-x+1} \gg \cdots \gg \nu_{i-1} \gg \nu_{i+1}+1 \gg \cdots \gg \nu_{j}+1$. We then obtain $\nu_{i-x}-x+1+i-j$, and since

$$
\nu_{i-x} \gg \nu_{i-x+1} \gg \cdots \gg \nu_{i-1} \gg \nu_{i},
$$

and $\nu_{i-x+1}, \ldots, \nu_{i-1} \in \mathcal{P}$, we have by (2.13) that $\nu_{i-x}-x+1 \gg \nu_{i}$, which is equivalent to $\nu_{i-x}-x+1+i-j \gg \nu_{i}-j+i$. We obtain by (2.19) that

$$
\begin{aligned}
& \text { either } \beta\left(\nu_{i-x}-x+1+i-j\right) \succ \alpha\left(\nu_{i}-j+i\right) \\
& \text { or } \alpha\left(\nu_{i}-j+i\right)+1 \gg \alpha\left(\nu_{i-x}-x+i-j\right) \succ \beta\left(\nu_{i-x}-x+i-j\right) \succ \beta\left(\nu_{i}-j+i\right) .
\end{aligned}
$$

In any case, the splitting in Step 2 occurs before $\beta\left(\nu_{i}-j+i\right)$. We set then

$$
\begin{aligned}
& \gamma^{2}=\nu_{1} \gg \cdots \gg \alpha\left(\nu_{i-x}-y\right), \\
& \mu^{2}=\beta\left(\nu_{i-x}-y\right) \succ \cdots \succ \beta\left(\nu_{i}-j+i\right) \succ \nu_{j+1} \succ \cdots \succ \nu_{t},
\end{aligned}
$$


where $y$ is the number of iterations of Step $\mathbf{1}$ before moving to Step 2, and by reasoning as before for the different cases, we can easily see that the conditions of Proposition 4.3 are respected. We obtain the result recursively. We also observe that the sequence $\gamma^{u}, \mu^{u}$ is exactly what we obtain by applying successively iteration of Step $\mathbf{1}$ and Step $\mathbf{2}$ of the transformation $\Phi$ on $\gamma^{u+1}, \mu^{u+1}$.

By the lemma, since the number of secondary parts decreases by one at each passage from Step 2 to Step 1, we will stop after exactly the number of secondary parts in $\nu$. And the result is of the form $\gamma^{U}, \mu^{U} \in \mathcal{O}$ with $\gamma^{U}$ well-ordered by $\gg$, and the last part of the first partition and the first of the second partition are consecutive in terms of $\succ$. We then conclude that $\Psi\left(\mathcal{E}_{1}\right) \subset \mathcal{O}$. Since all the steps are reversible by $\Phi$, we also have $\Phi \circ \Psi_{\mid \mathcal{E}_{1}}=I d_{\mathcal{E}_{1}}$.

\section{BiJective Proof of Theorem 1.5}

In this section, we will describe a bijection for Theorem 1.5. For brevity, we refer to the partitions in Theorem $\mathbf{1 . 5}$ as quaternary partitions.

5.1. From $\mathcal{E}_{1}$ to quaternary partitions. We consider the patterns $\left((k+1)_{a d}, k_{b c}\right),\left(k_{c d}, k_{a b}\right)$ and we sum them as follows:

$$
\begin{aligned}
(k+1)_{a d}+k_{b c} & =(2 k+1)_{a b c d} \\
k_{c d}+k_{a b} & =2 k_{a b c d} .
\end{aligned}
$$

Let us now take a partition $\nu$ in $\mathcal{E}_{1}$. We then identify all the patterns $\left(M^{i}, m^{i}\right) \in\{((k+$ $\left.\left.1)_{a d}, k_{b c}\right),\left(k_{c d}, k_{a b}\right)\right\}$ and suppose that

$$
\nu=\nu_{1}, \ldots, \nu_{x}, M^{1}, m^{1}, \nu_{x+1}, \ldots, \nu_{y}, M^{2}, m^{2}, \nu_{y+1}, \ldots, M^{t}, m^{t}, \ldots, \nu_{s} .
$$

As long as we have a pattern $\nu_{j}, M^{i}, m^{i}$, we cross the parts by replacing them using

$$
\nu_{j}, M^{i}, m^{i} \longmapsto M^{i}+1, m^{i}+1, \nu_{j}-2
$$

At the end of the process, we obtain a final sequence

$$
N^{1}, n^{1}, N^{2}, n^{2}, \ldots, N^{t}, n^{t}, \nu_{1}^{\prime}, \ldots, \nu_{s}^{\prime}
$$

Finally, the associated pair of partitions is set to be $\left(K^{1}, \ldots, K^{t}\right), \nu^{\prime}=\left(\nu_{1}^{\prime}, \ldots, \nu_{t}^{\prime}\right)$, where $K^{i}=N^{i}+n^{i}$ according to (5.1).

To sum up the previous transformation, we only remark that, for each quaternary part $K^{i}$ obtained by summing of the original pattern $M^{i}, m^{i}$, we add twice the number of the remaining primary and secondary parts in $\nu$ to the left of the pattern that gave $K^{i}$, while we subtract from these parts two times the number of quaternary parts obtained by patterns that occur to their right. 
With the example $11_{c}, \underline{10_{c d}, 10_{a b}}, 6_{d}, 5_{a b}, \underline{3_{a d}, 2_{b c}}, 1_{a}$,

\begin{tabular}{|c|c|c|c|c|c|c|c|c|c|c|}
\hline $10_{c d}$ & & $11_{c}$ & & $11_{c d}, 11_{a b}$ & & $11_{c d}, 11_{a b}$ & & $11_{c d}, 11_{a b}$ & & $11_{c d}, 11_{a b}$ \\
\hline $10_{a b}$ & & $10_{c d}, 10_{a b}$ & & $9_{c}$ & & $9_{c}$ & & $9_{c}$ & & $6_{a d}, 5_{b c}$ \\
\hline $\begin{array}{c}6_{d} \\
5_{a b}\end{array}$ & $\mapsto$ & $\begin{array}{c}6_{d} \\
5_{a b}\end{array}$ & $\mapsto$ & $\begin{array}{c}6_{d} \\
5_{a b}\end{array}$ & $\mapsto$ & $\begin{array}{c}6_{d} \\
4_{a d}, 3_{b c}\end{array}$ & $\mapsto$ & $\begin{array}{c}5_{a d}, 4_{b c} \\
4_{d}\end{array}$ & $\mapsto$ & $\begin{array}{l}7_{c} \\
4_{d}\end{array}$ \\
\hline $3_{a d}$ & & $3_{a d}, 2_{b c}$ & & $3_{a d}, 2_{b c}$ & & $3_{a b}$ & & $3_{a b}$ & & $3_{a b}$ \\
\hline $\begin{array}{l}2 b c \\
1\end{array}$ & & $1_{a}$ & & $1_{a}$ & & $1_{a}$ & & $1_{a}$ & & $1_{a}$ \\
\hline
\end{tabular}

we obtain $\left[\left(22_{a b c d}, 11_{a b c d}\right),\left(7_{c}, 4_{d}, 3_{a b}, 1_{a}\right)\right]$. We now proceed to show that the image of this mapping is indeed a quaternary partition. The inverse mapping will be presented in the next subsection.

(1) Quaternary parts are well-ordered. Let us consider two consecutive patterns $\left(M^{j}, m^{j}\right)=\left(k_{p}, l_{q}\right)$ and $\left(M^{j+1}, m^{j+1}\right)=\left(k_{p^{\prime}}^{\prime}, l_{q^{\prime}}^{\prime}\right)$. Since $\nu$ is well-ordered by $\gg$, we have by (2.13) and (2.10) that

$$
l_{q} \triangleright l_{p_{1}}^{1} \triangleright \cdots \triangleright l_{p_{i}}^{i} \triangleright k_{p^{\prime}}^{\prime} .
$$

By (2.10), we then have that $l_{q} \succ k_{p^{\prime}}^{\prime}+i+1$ so that $l-k^{\prime} \geq i+1+\chi\left(q \leq p^{\prime}\right)$. Since by (2.13),$k-l=\chi(p \leq q)$ and $k^{\prime}-l^{\prime}=\chi\left(p^{\prime} \leq q^{\prime}\right)$, we then have that

$$
\begin{aligned}
k+l-\left(k^{\prime}+l^{\prime}\right) & =\chi(p \leq q)+\chi\left(p^{\prime} \leq q^{\prime}\right)+2\left(l-k^{\prime}\right) \\
& \geq \chi(p \leq q)+\chi\left(p^{\prime} \leq q^{\prime}\right)+2 \chi\left(q \leq p^{\prime}\right)+2 i+2
\end{aligned}
$$

and we obtain that

$$
\begin{aligned}
& \chi(c d \leq a b)+\chi(c d \leq a b)+2 \chi(a b \leq c d)=2 \\
& \chi(c d \leq a b)+\chi(a d \leq b c)+2 \chi(a b \leq a d)=3 \\
& \chi(a d \leq b c)+\chi(c d \leq a b)+2 \chi(b c \leq c d)=3 \\
& \chi(a d \leq b c)+\chi(a d \leq b c)+2 \chi(b c \leq a d)=2,
\end{aligned}
$$

so that $k+l-\left(k^{\prime}+l^{\prime}\right) \geq 4+2 i$. We will then have, after adding twice the remaining primary and secondary elements to their left, that the difference between two consecutive quaternary parts will be at least 4 .

(2) The partition $\nu^{\prime}$ is in $\mathcal{E}_{2}$. Let us consider two consecutive elements $\nu_{x}=k_{p}, \nu_{x+1}=$ $l_{q}$. We then have for consecutive patterns $M^{u}, m^{u}$ between $k_{p}$ and $l_{q}$ that

$$
k_{p} \triangleright M^{i} \gg m^{i} \gg \cdots \gg M^{j} \gg m^{j} \triangleright l_{q} \text {. }
$$

For the case $\left(M^{j}, m^{j}, l_{q}\right) \neq\left(3_{a d}, 2_{b c}, 1_{a}\right)$, since by Lemma 2.3. $M^{u} \succeq M^{u+1}+2$, $M^{j} \succeq l_{q}+2$, and by (2.13), we have that $k_{p} \succ M^{i}+1$, and then

$$
k_{p} \succ 1+2(j-i+1)+l_{q} \Longrightarrow k_{p} \triangleright 2(j-i+1)+l_{q} \cdot
$$

For the case $\left(M^{j}, m^{j}, l_{q}\right)=\left(3_{a d}, 2_{b c}, 1_{a}\right)$, we obtain that

$$
k_{p}-2(j-i+1)+1 \succ 3_{a d}
$$

and this means that $k_{p}-2(j-i+1)+1 \succeq 3_{a}$ so that $k_{p}-2(j-i+1) \succeq 2_{a} \triangleright 1_{a}$. 
In any case, $k_{p} \triangleright 2(j-i+1)+l_{p}$, and this implies that after the subtraction of twice the number of the quaternary parts obtained to their right, these parts will be well-ordered by $\triangleright$.

(3) The minimal quaternary part is well-bounded. Let us first suppose that the tail of $\nu$ consists only of patterns $M^{u}, m^{u}$. We then have that

$$
\nu_{s} \triangleright M^{i} \gg m^{i} \gg \cdots \gg M^{t} \gg m^{t}
$$

and, by Lemma 2.3 and (2.13),$\nu_{s}-2(t-i+1)+1 \succeq M^{t} \succeq 2_{c d}$, so that $\nu_{s}^{\prime}=\nu_{s}-2(t-i+1) \succeq 1_{c d} \succ 1_{a}$. This means that $1_{a} \notin \nu^{\prime}$. We also obtain that $K^{t}=M^{t}+m^{t}+2 s \geq 2 s+4$.

Now suppose that the tail of $\nu$ has the form

$$
l_{q} \triangleright \nu_{u} \triangleright \cdots \triangleright \nu_{s}
$$

with $M^{t}, m^{t}=k_{p}, l_{q}$. By (2.10), we obtain that $l_{q} \succ \nu_{s}+s-u+1$.

- If $\nu_{s}=1_{a}$, we then have

$$
\begin{aligned}
k+l & =\chi(p \leq q)+2 l \\
& \geq \chi(p \leq q)+2(s-u+2+\chi(q \leq a)) \\
& =2(s-u+1)+2+\chi(p \leq q)+2 \chi(q \leq a),
\end{aligned}
$$

and with $(p, q) \in\{(a d, b c),(c d, a b)\}$ we have

$$
\begin{aligned}
& \chi(a d \leq b c)+2 \chi(b c \leq a)=1 \\
& \chi(c d \leq a b)+2 \chi(a b \leq a)=2
\end{aligned}
$$

so that $k+l \geq 2(s-u+1)+3$. Then after the addition of $2(u-1)$ for the remaining primary and secondary parts of $\nu$ to the left of the pattern $\left(M^{t}, m^{t}\right)$, we obtain that the smallest quaternary part is at least $2 s+3$. Note that $\nu_{s}^{\prime}=\nu_{s}=1_{a}$.

- When $\nu_{s}=h_{r} \neq 1_{a}$, we obtain that

$$
\begin{aligned}
k+l & \geq \chi(p \leq q)+2(s-u+1+h+\chi(q \leq r)) \\
& =2(s-u+1)+2 h+\chi(p \leq q)+2 \chi(q \leq r),
\end{aligned}
$$

so that if $h \geq 2$, then $k+l \geq 2(s-u+1)+4$. If not, $h=1$, and since there is no secondary part of size 1 , we necessary have that $r \geq b$, so that $\chi(q \leq r)=1$ whenever $q \in\{a b, b c\}$. We thus obtain $k+l \geq 2(s-u+1)+4$. We then conclude that for $\nu_{s} \neq 1_{a}$, the smallest quaternary part is at least $2 s+4$.

In any case, we have that the smallest quaternary part is at least $2 s+4-\chi\left(1_{a} \in \nu^{\prime}\right)$.

5.2. From quaternary partitions to $\mathcal{E}_{1}$. Recall by (5.1) that $K_{a b c d}$ splits as follows :

$$
\begin{aligned}
(k+1)_{a d}+k_{b c} & =(2 k+1)_{a b c d} \\
k_{c d}+k_{a b} & =2 k_{a b c d}
\end{aligned}
$$


Let us then consider partitions $\left(K^{1}, \ldots, K^{t}\right)$ and $\nu=\left(\nu_{1}, \ldots, \nu_{s}\right) \in \mathcal{E}_{2}$, with quaternary part $K^{u}$ such that $K^{t} \geq 4+2 s-\chi\left(1_{a} \in \nu\right)$ and $K^{u}-K^{u+1} \geq 4$. We also set $K^{u}=\left(k^{u}, l^{u}\right)$ the decomposotion according to (5.1). We then proceed as follows by beginning with $K^{t}$ and $\nu_{1}$,

Step 1: If we do not encounter $K^{u+1}=\left(k^{u+1}, l^{u+1}\right)$ and $\nu_{i} \neq 1_{a}$ and $\nu_{i}+2 \triangleright k^{u}-1$, then replace

$$
\begin{aligned}
\nu_{i} & \longmapsto \nu_{i}+2 \\
\left(k^{u}, l^{u}\right) & \longmapsto\left(k^{u}-1, l^{u}-1\right)
\end{aligned}
$$

and move to $i+1$ and redo Step $\mathbf{1}$. Otherwise, move to Step 2.

Step 2 If we encounter $K^{u+1}=k^{u+1} \gg l^{u+1}$, then split $\left(k^{u}, l^{u}\right)$ into $k^{u} \gg l^{u}$. If not, it means that we have met $\nu_{i}$ such that $\nu_{i}+2 \not k^{u}-1$. Then we split $k^{u} \gg l^{u}$. Since we have $\nu_{i}+2 \not k^{u}-1$, which is equivalent by (2.10) to $k^{u} \succeq \nu_{i}+2$, by Lemma 2.3, this is exactly the condition to avoid the forbidden patterns, with $k^{u} \gg l^{u} \triangleright \nu_{i}$.

We can now move to Step 1 with $u-1$ and $i=1$.

\begin{tabular}{|c|c|c|c|c|c|c|c|c|c|c|c|c|}
\hline $\begin{array}{c}11_{c d}, 11_{a b} \\
6_{a d}, 5_{b c} \\
7_{c} \\
4_{d} \\
3_{a b} \\
1_{a}\end{array}$ & $\mapsto$ & $\begin{array}{c}11_{c d}, 11_{a b} \\
9_{c} \\
5_{a d}, 4_{b c} \\
4_{d} \\
3_{a b} \\
1_{a}\end{array}$ & $\mapsto$ & $\begin{array}{c}11_{c d}, 11_{a b} \\
9_{c} \\
6_{d} \\
4_{a d}, 3_{b c} \\
3_{a b} \\
1_{a}\end{array}$ & $\mapsto$ & $\begin{array}{c}11_{c d}, 11_{a b} \\
9_{c} \\
6_{d} \\
5_{a b} \\
3_{a d}, 2_{b c} \\
1_{a}\end{array}$ & $\mapsto$ & $\begin{array}{c}11_{c d}, 11_{a b} \\
9_{c} \\
6_{d} \\
5_{a b} \\
3_{a d} \\
2_{b c} \\
1_{a}\end{array}$ & $\mapsto$ & $\begin{array}{c}11_{c} \\
10_{c d}, 10_{a b} \\
6_{d} \\
5_{a b} \\
3_{a d} \\
2_{b c} \\
1_{a}\end{array}$ & $\mapsto$ & $\begin{array}{c}11_{c} \\
10_{c d} \\
10_{a b} \\
6_{d} \\
5_{a b} \\
3_{a d} \\
2_{b c} \\
1_{a}\end{array}$ \\
\hline
\end{tabular}

With the example $\left[\left(22_{a b c d}, 11_{a b c d}\right),\left(7_{c}, 4_{d}, 3_{a b}, 1_{a}\right)\right]$, we obtain

It is easy to check that when two quaternary parts meet in Step 2, we will always have $l^{u} \gg k^{u+1}$, since this is exactly the condition for the minimal difference $K^{u}-K^{u+1} \geq 4$ and they crossed the same number of $\nu_{i}$. We can also check that even if the minimal part crossed $\nu_{1}, \ldots, \nu_{s} \neq 1_{a}$, we will still have at the end $K^{t} \geq 4$ and for $\nu_{s}=1_{a}, K^{t} \geq 5$. We see with (5.1) that the size of $m^{t}$ is at least equal to 2 , and for the case $\nu_{s}=1_{a}, m^{t}$ is at least equal to $2_{b c} \gg 1_{a}$. The partition obtained is then in $\mathcal{E}_{1}$.

\section{REFERENCES}

[1] K. ALLADI, G.E. ANDREWS and A. BERKOVICH, A new four parameter q-series identity and its partitions implications, Invent. Math. 153 (2003), 231-260.

[2] K. ALLADI, G.E. ANDREWS and B. GORDON, Generalizations and refinements of a partition theorem of Göllnitz, J. Reine Angew. Math. 460 (1995), 165-188.

[3] K. ALLADI and B. GORDON, Generalization of Schur's partition theorem, Manuscripta Math. 79 (1993), 113-126.

[4] G.E. ANDREWS, A new generalization of Schur's second partition theorem, Acta Arith. 14 (1968), 429-434.

[5] G.E. ANDREWS, On a partition theorem of Göllnitz and related formula, J. Reine Angew. Math. 236 (1969), 37-42.

[6] D. BRESSOUD, A combinatorial proof of Schur's 1926 partition theorem, Proc. Amer. Math. Soc. $\mathbf{7 9}$ (1980), 338-340.

[7] H. GÖLLNITZ, Partitionen mit Differenzenbedingungen, J. Reine Angew. Math. 225 (1967), 154-190.

[8] PADMAVATHAMMA, M. RUDY SALESTINA and S.R. SUDARSHAN, Combinatorial proof of the Göllnitz's theorem on partitions, Adv. Stud. Contemp. Math. 8 (2004), no.1, 47-54. 
[9] I. SCHUR, Zur additiven zahlentheorie, Sitzungsberichte der Preussischen Akademie der Wissenschaften (1926), 488-495.

[10] J.Y.J ZHAO, A bijective proof of the Alladi-Andrews-Gordon partition theorem, Electron. J. Combin. 22 (2015), no. 1, Paper 1.68

IRIF, Université de Paris, Bâtiment Sophie Germain, Case courrier 7014, 8 Place Aurélie Nemours, 75205 Paris Cedex 13, France

Email address: konan@irif.fr 